2019-08-24

\title{
De-Noising Signals using Wavelet Transform in Internet of Underwater Things
}

\author{
Khan, Asiya
}

http://hdl.handle.net/10026.1/13283

\subsection{7/978-3-030-29513-4_85}

Springer series "Advances in Intelligent Systems and Computing"

Springer International Publishing

All content in PEARL is protected by copyright law. Author manuscripts are made available in accordance with publisher policies. Please cite only the published version using the details provided on the item record or document. In the absence of an open licence (e.g. Creative Commons), permissions for further reuse of content should be sought from the publisher or author. 


\title{
De-Noising Signals using Wavelet Transform in Internet of Underwater Things
}

\author{
Asiya Khan*, Richard Pemberton, Abdul Momen and Daniel Bristow \\ University of Plymouth, Plymouth, PL4 8AA, UK \\ *Corresponding author asiya.khaneplymouth.ac.uk
}

\begin{abstract}
Internet of Underwater Things (IoUT) is an emerging field within Internet of Things (IoT) towards smart cities. IoUT has applications in monitoring underwater structures as well as marine life. This paper presents preliminary work where sensor nodes were built on Arduino Uno platform with temperature and pressure sensors with wireless capability. The sensors nodes were then tested in the Flumes of the COAST laboratory to determine the maximum depth achievable in fresh water before the signal is lost as radio frequencies are susceptible to interference under water. Further, the received signals were de-noised using Wavelet Transform, Daubechies thresholding techniques at level 5. Preliminary results suggests that at a depth of $30 \mathrm{~cm}$, signal was lost, de-noising of the signal was achieved with very small errors (a mean squared error of 0.106 and 0.000446 and Peak-Sign-to-Noise Ratios of $70.18 \mathrm{~dB}$ and $58.83 \mathrm{~dB}$ for the pressure and temperature signals respectively. Results from this study will lay the foundation to further investigations in wireless sensor networks in IoUT integrating the de-noising techniques.
\end{abstract}

Keywords: IoUT, Sensors, Arduino Uno, Wavelet Transform.

\section{Introduction}

IoUT was first introduced in [1] and is defined as "the network of smart interconnected underwater objects". The smart objects could be different types of underwater sensors, autonomous underwater/surface vehicles (AUVs/ASVs), buoys, ships, to name a few. IoUT is a relatively new class of Internet of Things (IoT) and will be an enabler for numerous applications within the roadmap towards 'smart city'.

Over $70 \%$ of earth is underwater and majority of these areas are not explored. Also, there is a steady increase in investment in offshore renewable energy e.g. wave energy converters or floating wind, there is an increasing need to monitor these devices with sensors that can work both on the surface and underwater. These sensor nodes are small, battery operated and built on microcontrollers with some wireless capability. The communication between the sensors has given rise to IoUT and Underwater Wireless Sensor Networks (UWSNs) as a promising network system.

A number of researchers have presented their works on the challenges in routing protocols [2], [3], channel models [4] and environmental monitoring [5]. Sensors are the main components of UWSNs. Sensors are the nodes with wireless capability (based 
on radio/sound frequency) distributed in the shallow or deep water and have the capability to sense, relay, and transmit the data to the surface of the water, called sink(s). Sinks are the nodes with both acoustic and radio modems. When data arrive at sinks (through acoustic/radio channels), the sinks will forward data to the remote monitoring centre (through radio channels) on the sea-shore where the received data is collected and analyzed as shown in Fig. 1.

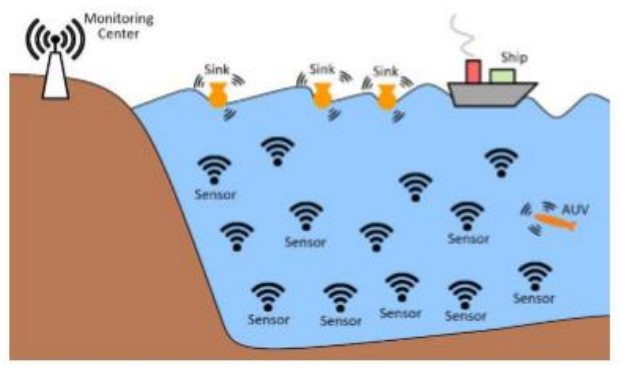

Fig. 1. UWSN architecture in IoUT [4]

This paper presents preliminary work from sensor nodes built on Arduino Uno, an open source microcontroller with temperature and pressure sensors and a $433 \mathrm{MHz}$ wireless transmitter receiver for IoUT. The sensor nodes were tested in the Flumes of the University of Plymouth COAST Laboratory at varying depths. Therefore, the main contributions of the paper are twofold - firstly to determine the maximum depth beyond which radio signals are lost in IoUT and secondly to use Wavelet Transform to de-noise the received signal.

The rest of the paper is organized as follows. Section 2 describes the problem statement, whereas, the method is presented in Section 3. Section 4 presents the results and Section 5 concludes the paper.

\section{Problem Statement}

Underwater communication mainly uses acoustic communication. Acoustic communication has medium range, low bandwidth and poor performance in shallow water as it is affected by noise. Another method of underwater communication is utilizing the optical channel which has ultra-high bandwidth over short distances but is affected by turbidity, light and marine fouling and also has low performance between water-to-air boundary.

Hence, the motivation to investigate radio frequencies underwater, however, they are susceptible to interference. Some of the challenges of IoUT include delay, reliability, bandwidth, power and movement of sensors due to water currents. In shallow waters, acoustic signals experience multi-path propagation, reverberation and ambient noise and impact on the marine life negatively [6], whereas, optical signals suffer from high absorption and backscatter [7]. Therefore, IoUT based on radio frequencies can offer higher bandwidth and better transmission especially on the boundaries i.e. water-air, seabed-ice and have applications in water quality monitoring, 
environmental monitoring, underwater exploration on autonomous surface/underwater vehicles.

Therefore this paper aims to address the following research question - what is the maximum depth for data to be received in IoUT? Further, to apply techniques from machine learning to de-noise the data. This will allow us to implement machine learning techniques in real time to get meaningful information from the data received from IoUT monitoring applications.

\section{$3 \quad$ Method}

In shallow waters radio frequencies have several advantages over acoustic waves, as they can provide faster and more efficient communication between network nodes with higher data rates. However, because radio frequencies are propagated differently depending on the type of water e.g. salty water vs fresh water, it can have an effect on the communication of data.

To address the research question, sensor nodes were built on Arduino Uno, an open source platform. The sensors chosen were temperature (accuracy of $0.8^{\circ} \mathrm{C}$ ) and pressure (accuracy of 20mbar) was the Ms5803-14Ba, whereas, for wireless communication the LPD4333 MHz (Low power Device) transmitter/receiver was chosen with a power source of $9 \mathrm{~V}$ DC alkaline battery. The system is shown in Fig. 2a. The system was then water proofed. Fig. $2 \mathrm{~b}$ shows the $\mathrm{CAD}$ design of the water proof housing.

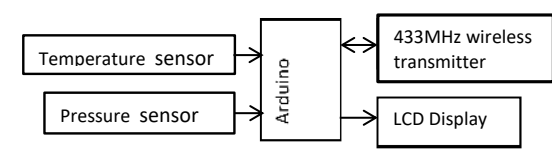

Fig. 2a. IoUT sensor nodes

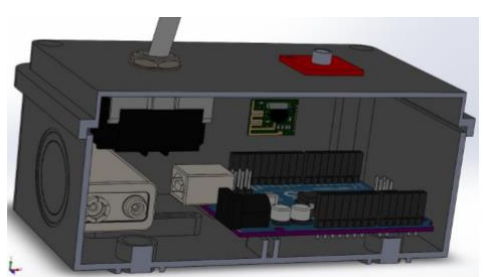

Fig 2b. Water proof housing for the IoUT sensors

The system was then tested in the $20 \mathrm{~m}$ Flumes in the COAST laboratory at the University of Plymouth over three days. Tests were conducted at various depths to

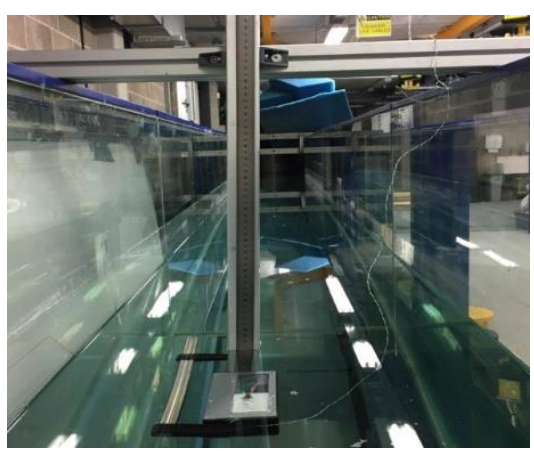

Fig. 3a. Test setup of the sensor nodes in the Flumes

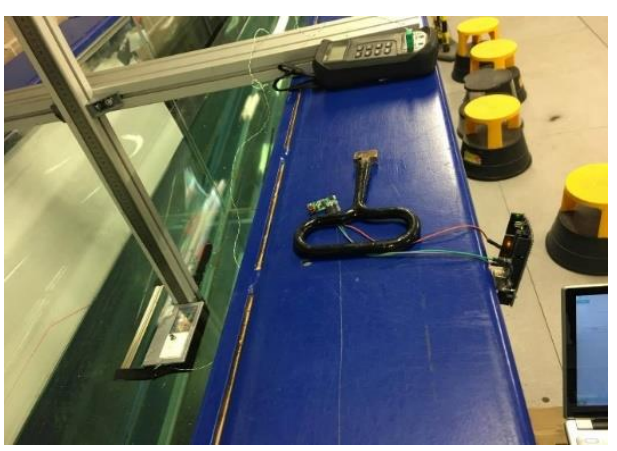

Fig. 3b. Receiver set-up held in place by a weight and connected to the laptop 
determine the maximum depth in fresh water before packets are lost. The set-up is shown in Figs. $3 a$ and $b$.

A control test was carried out to check for sensor accuracy. Average temperature reading was fairly close to the temperature received by the sensors, however, the average pressure reading was adjusted to produce the corrected average water pressure according to eq. (1).

$$
\text { Ptotal }=\text { Patm }+ \text { Pfluid }
$$

The data received was noisy. In real time applications, often there is noise with the data, therefore, it is important to use techniques to de-noise the data and hence get meaningful information. The sensor data received were then de-noised using machine learning technique of wavelet analysis in MATLAB. Wavelet Transform is a technique that decomposes a signal into different frequency level of coefficients. The signals are then analyzed in different frequency bands and scales can be helpful determining signals behaviors' in different sections of frequency. This allows for detecting the corruption within the signal and eliminating it becomes a relatively easy process. There are many different types of wavelets, we chose the Daubechies wavelet [6] as it has shown better results from literature.

\section{$4 \quad$ Results}

The temperature and pressure readings at different depths are shown in Figs. 4a and 4b. The depth was varied in $5 \mathrm{~cm}$ steps up to $30 \mathrm{~cm}$ at which points most packets were lost.

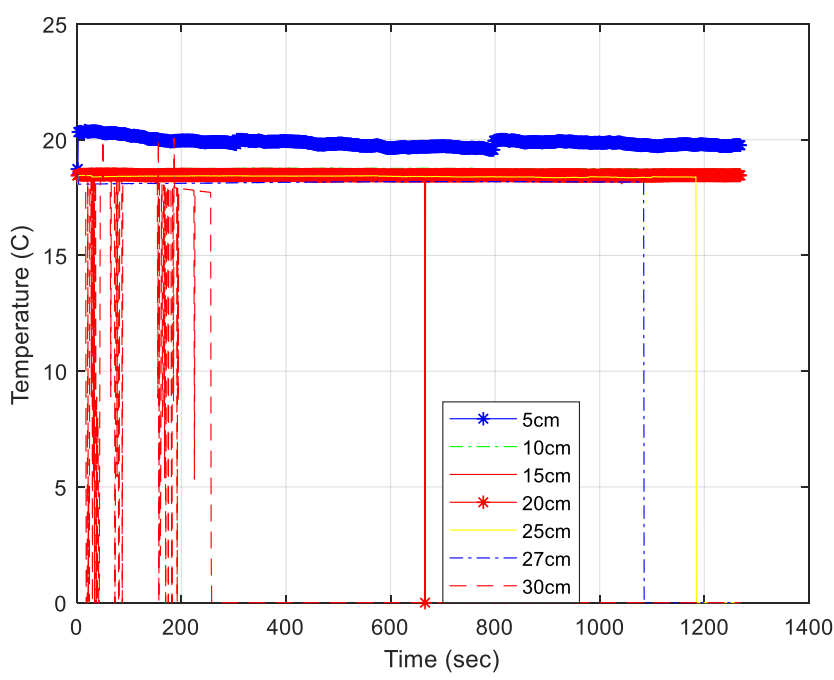

Fig. 4a. Temperature over 20 minutes for varying depths 


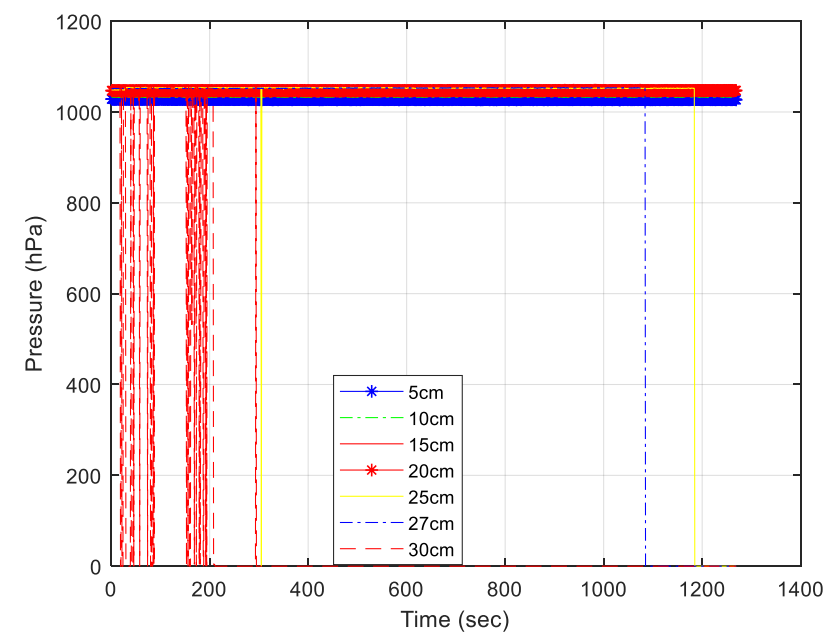

Fig. 4b. Pressure over 20 minutes for varying depths

Fig. 5 shows the packet loss rate at the different depths. It shows that at a depth of $30 \mathrm{~cm}$ all packets were lost, whereas, for a depth of $27 \mathrm{~cm}$ almost $80 \%$ of packet were lost.

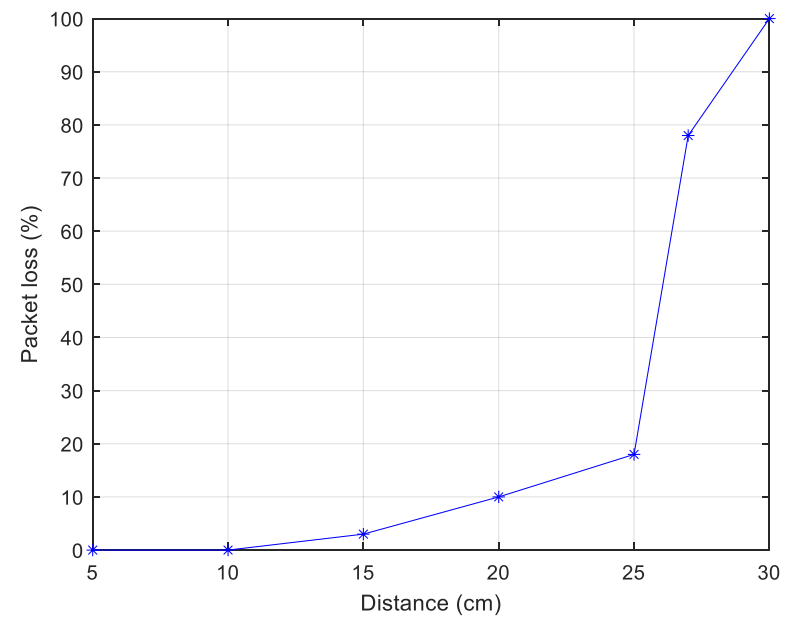

Fig. 5. The sensor data received at different depths

The sensor data was then de-noised using the Wavelet Transform in MATLAB. Figs. $6 \mathrm{a}$ and $\mathrm{b}$ show the original pressure and temperature sensor data versus the de-noised data. The first step to eliminate the noise in the signal involves the decomposition of signal into approximation and detail coefficients. We chose five level Daubechies wavelet. We got a mean squared error of 0.106 and 0.000446 and Peak-Signal-to-Noise Ratios (PSNR) of $70.18 \mathrm{~dB}$ and $58.83 \mathrm{~dB}$ for the pressure and temperature de-noised signals respectively. 


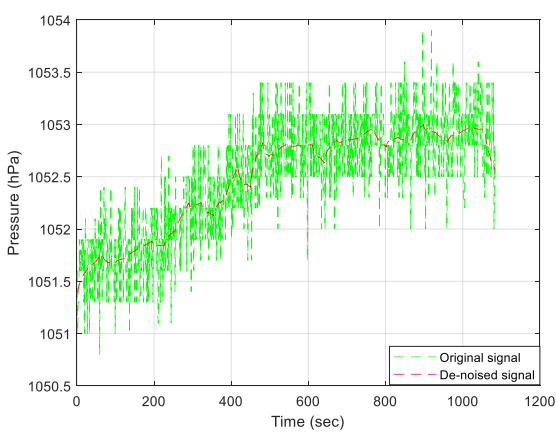

Fig. 6a. Original pressure signal versus the denoised signal at $25 \mathrm{~cm}$ depth

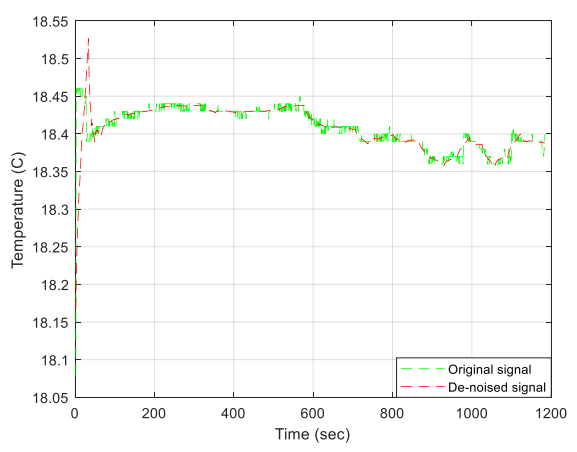

Fig. 6b. Original temperature signal versus the de-noised signal at $25 \mathrm{~cm}$ depth

\section{Conclusions}

This paper presents preliminary results on IoUT sensor nodes, built and tested in the Coast laboratory. Our study found that up to a depth of $25 \mathrm{~cm}$ radio frequency signals were received on shore from sensors nodes built on Arduino Uno with temperature and pressure sensors. We also were able to de-noise the signals using Wavelet Transform with mean very low mean square error and good PSNR.

Our future work will establish UWSN for IoUT and test under different wave conditions as well as in salty water.

\section{References}

1. Domingo, M., C., "An overview of the internet of underwater things", Journal of Network and Computer Applications, vol. 35, no. 6, (2012).

2. Liou, E-C, Kao, C-C, Chang, C-H, Lin Y-S and Huang, C-J, "Internet of underwater things: challenges and routing protocols", Proceedings of IEEE International Conferences on Applied System innovation, IEEE ICASI 2018-Meen, Prior \& Lam (Eds), (2018).

3. Z. Zhou, B. Yao, R. Xing, L. Shu, and S. Bu, "E-CARP: an energy efficient routing protocol for UWSNs in the internet of underwater things", IEEE Sensors Journal, vol. 16, no. 11, (2016).

4. Kao, C-C, Lin, Y-S, Wu, G-D and Huang, C-J, "Study of Applications, Challenges, and Channel Models on the Internet of Underwater", Proceedings of IEEE International Conferences on Applied System innovation, IEEE ICASI 2018-Meen, Prior \& Lam (Eds), (2017).

5. Fang, S., Xu, L.D., Zhu, Y., Ahati, J., Pei, H., Yan, J. and Liu, Z., "An integrated system for regional environmental monitoring and management based on internet of things", IEEE Transactions on Industrial Informatics, vol. 10, no. 2, (2014).

6. Jepson, P., Arbelo, M., Deavile, R., Patterson, I., Castro, P., Baker, J., Degollada, E., Ross, H., Herraez, P., Pocknell et al., Gas-bubble lessions in stranded cetaceans, Nature, vol. 425, no. 6958 , pp. $575-576,2003$ 
7. Wang, H., Yang, K., Zheng, K., Han, Y. and Xiao, P., Experimental investigation on electromagnetic wave propagation across sea-to-air interface, IEEE Oceans, Taipei, Taiwan, April 2014, pp1-6.

8. Dautov, C., P. and Ozerdem, M., S., "Wavelet transform and signal de-noising using wavelet method", $26^{\text {th }}$ Signal Processing and Communications Applications Conference (SIU), 3-5 May Izmir, Turkey (2018). 